\title{
Dynamic Cooperative Glycan Assembly Blocks Binding of Bacterial Lectins to Epithelial Cells
}

\author{
Takuya Machida, Alexandre Novoa, Émilie Gillon, Shuangshuang Zheng, Julie Claudinon, Thorsten \\ Eierhoff, Anne Imberty, Winfried Römer, Nicolas Winssinger *[a]
}

\begin{abstract}
Pathogens frequently rely on lectins for adhesion and cellular entry into the host. Since these interactions typically result from multimeric binding of lectins to cell surface glycans, novel therapeutic strategies are being developed with the use of glycomimetics as competitors of such interactions. Herein we study the benefit of nucleic acid-based oligomeric assemblies with PNAfucose conjugates. We demonstrate that the interactions of a lectin with epithelial cells can be inhibited with conjugates that do not form stable assemblies in solution but benefit from the cooperativity of ligand-protein interactions and PNA hybridization to achieve high affinity. A dynamic dimeric assembly fully blocked the binding of the fucose-binding lectin BambL of Burkholderia ambifaria, a pathogenic bacterium, to epithelial cells with an efficiency of more than 700 fold compared to L-fucose.
\end{abstract}

The role of multivalent interactions at cellular interfaces is well recognized..$^{[1]}$ Frequently, these interactions involve cell surface glycans which are specifically recognized by protein receptors such as lectins. Such interactions are generally of low affinities but, a high overall avidity is achieved through mutivalency. Pathogens frequently harness the specific recognition of host epithelial glycans for adhesion and infection with multivalent lectins $^{[2-3]}$, and inhibition of these interactions by competition with soluble glycocompounds represents a promising therapeutic avenue. ${ }^{[4]}$ Herein, we investigated the gain of affinity achieved with nucleic acid-based assemblies oligomerizing L-fucose, and we demonstrate that the hybridization of dynamic fucose assembly operates cooperatively with ligand binding.

The Burkholderia cepacia complex is a group of closely related bacteria that cause lung infections in immunocompromised patients as well as in patients with

[a] T. Machida, Dr. A. Novoa, Prof. N. Winssinger

Department of Organic Chemistry, NCCR Chemical Biology

Faculty of Science, University of Geneva

Quai Ernest Ansermet 30, 1211 Geneva, Switzerland

E-mail: nicolas.winssinger@unige.ch

E. Gillon, Dr. A. Imberty

CERMAV UPR5301, CNRS, and Université Grenoble Alpes, BP 53

38041 Grenoble cedex 9, France

E-mail: anne.imberty@cermav.cnrs.fr

S. Zheng, Dr. J. Claudinon, Dr. T. Eierhoff, Prof. W. Römer

Faculty of Biology, Albert-Ludwigs-University Freiburg,

Schänzlestraße 1, and Centre for Biological Signalling Studies (BIOSS), Albert-Ludwigs-University Freiburg, Schänzlestraße 18 79104 Freiburg, Germany; present address of Dr. T. Eierhoff: Institute of Biochemistry, Heinrich-Heine-University Düsseldorf, Universitätsstraße 1, 40225 Düsseldorf, Germany

Email: winfried.roemer@bioss.uni-freiburg.de

Supporting information for this article is given via a link at the end of the document. granulomatous disease or cystic fibrosis. ${ }^{[5]}$ Burkholderia ambifaria lectin (BambL) and Ralstonia solanacearum lectin (RSL) are bacterial lectins that share a six-bladed beta-propeller fold formed by trimerization of monomers carrying two fucosebinding sites, resulting in six fucose binding sites on the same face (Figure 1). ${ }^{[5-6]}$ It has been shown that the interaction of RSL

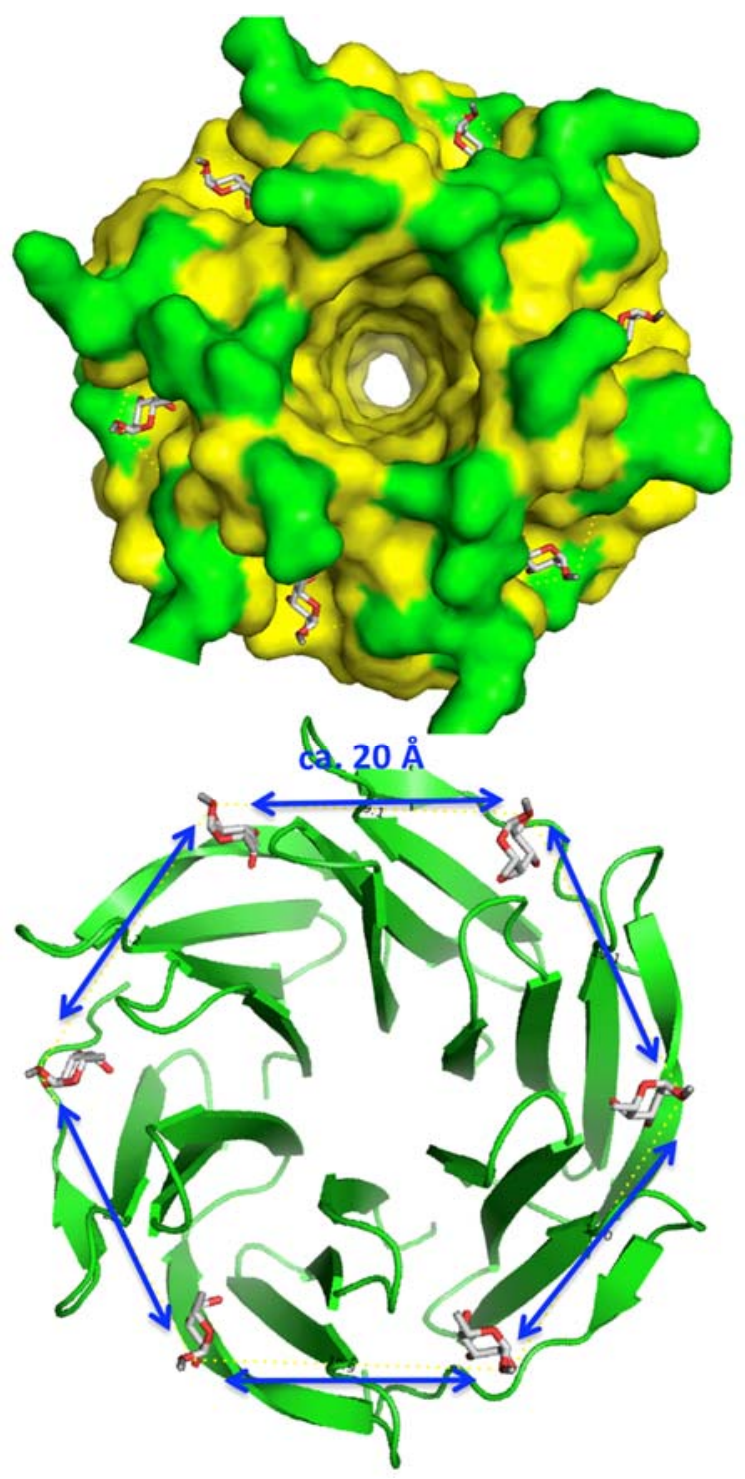

Figure 1. Space filling and ribbon representation of RSL crystal structure complexed with $\alpha$-methyl-L-fucoside represented as wire frame (PDB code 2BT9). Blue arrows indicate the $20 \AA$ through-space distance between the binding sites. RSL: Ralstonia solanacearum lectin. 
with fucosylated epitopes such as histo-blood group oligosaccharides exposed from glycolipids is sufficient to induce membrane invaginations in giant liposomes. ${ }^{[7-8]}$ Furthermore, oligomeric interactions are important for this invagination, since mutated lectins bearing only three fucose binding sites (e.g. R17A mutant) were also interacting with liposomes but failed to yield invagination. Based on the interest in using nucleic acid hybridization of glycan-PNA conjugates (PNA: peptide nucleic acid $)^{[9-16]}$ to tailor assemblies that can bridge multiple binding sites in target proteins, we sought to evaluate the benefit of oligomeric assemblies for RSL and ultimately, to outcompete the interaction of such lectins with epithelial cells.

We began our investigation with RSL, designing a fucose ligand flanked by two 8-mer PNA (Figure 2). Using the appropriate complementary sequences, the fucose ligand can be oligomerized incrementally to a cyclic hexamer that would form a ring around the protein. The 8-mer PNA duplex stretches over $34 \AA$ with a small curvature (though-space distance: PDB ID 3PA0) $)^{[17]}$ thus yielding a hexagone with a perimeter of $204 \AA$ which was deemed sufficiently large to fit the protein. The fucose ligand is linked to each side of the PNA with a $10 \AA$ flexible PEG spacer that leave some plasticity to fit the multivalent interaction. The 8-mer PNA have Tm > $60{ }^{\circ} \mathrm{C}$ thus yielding stable assemblies at room temperature. The affinities of the different assemblies were measured by surface plasmon resonance (SPR) using a chip with low protein loading to avoid artifact from assemblies bridging two proteins. The control L-fucose-triazole conjugate (Fuc-triazole, Figure 1) has an affinity for RSL of approx. $2 \mu \mathrm{M}$, which is in the same range that the one previously reported for fucose. ${ }^{[6]}$ The presence of PNA on both sides of fucose increases the affinity for the lectin by one order of magnitude compared to the Fuctriazole control compound resulting in a $K_{\mathrm{D}}$ of $362 \mathrm{nM}$, however, the PNA duplex lacking fucose did not show measurable affinity to the lectin. As shown in Figure 2, a significant gain in affinity is obtained in the progression from monomeric ligand with flanking double strand PNAs (362 $\mathrm{nM}$ ) to the dimeric one $(3.3 \mathrm{nM})$. This gain in affinity is consistent with the chelate effect of divalent interactions and confirmed that the 8-mer PNA with the resonance. flexible PEG linker is able to bridge two binding sites. However, progression to higher order oligomers only brought small incremental benefit ( $3 \mathrm{nM}$ for the trimer, $1 \mathrm{nM}$ for the tetramer, 0.9 $\mathrm{nM}$ for the pentamer and $0.5 \mathrm{nM}$ for the cyclic hexamer). Comparison of SPR sensorgrams obtained with monomeric and hexameric compounds is displayed in Figure 2 (See figure S1 for complete set of sensorgrams), illustrating the strong gain in affinity observed for oligomers. Enthalpy-entropy compensations have frequently been attributed to diminishing return in affinity
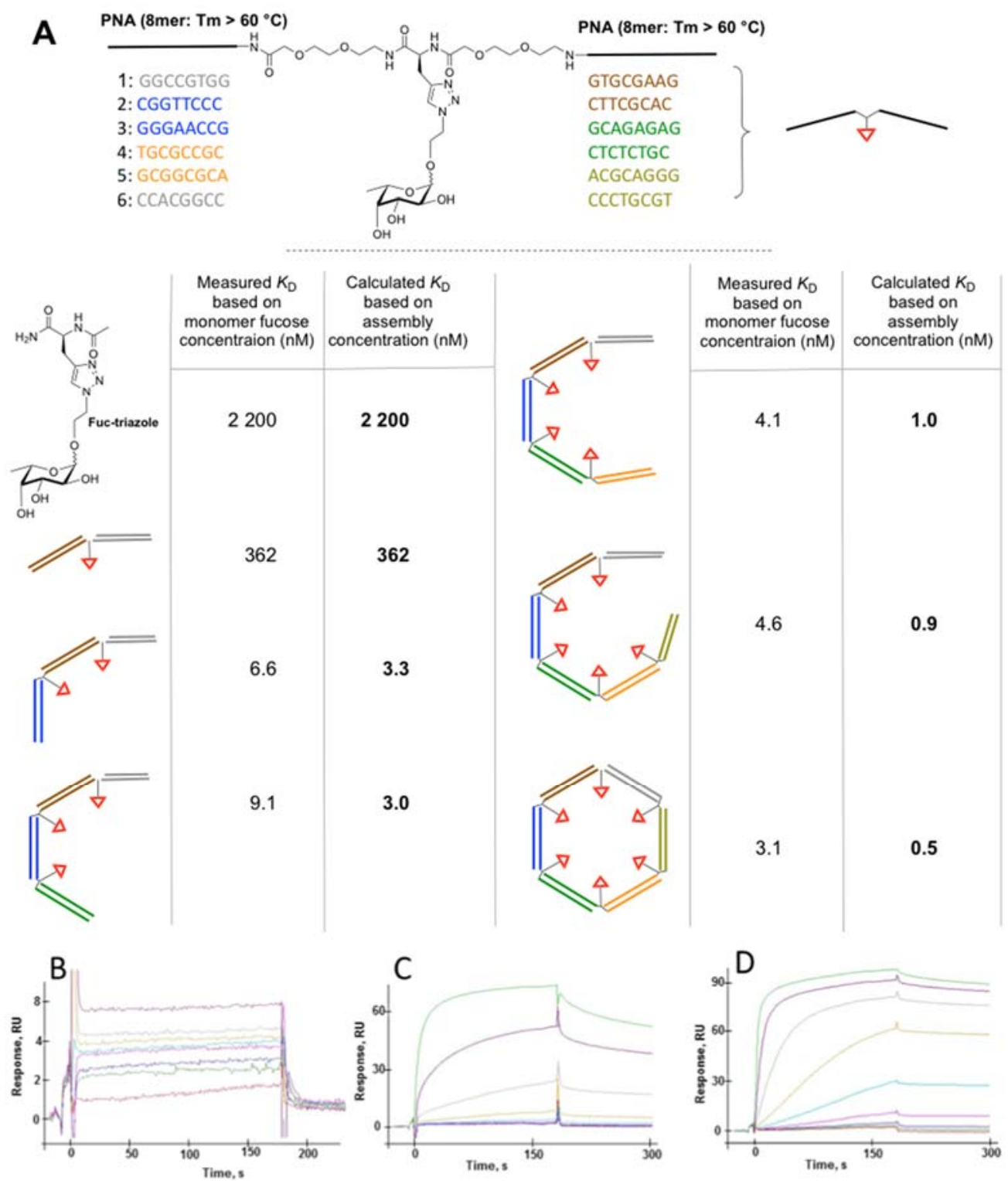

Figure 2. A: Structure of the PNA-glycan adduct (top, PNA color denotes self-complementary) and SPR-measured $K_{D}$ for the assemblies. B: SPR sensorgrams for Fuc-triazole injected on RSL chips with concentrations starting at $3.33 \mu \mathrm{M}$ and diluted by 3-fold at each step. C: Same experiments with the assembly produced from the mixture of compound 1 and 2 forming a dimer (starting concentration $1 \mu \mathrm{M}$ ). D: Same with compounds 1 to 6 that form hexavalent assembly (starting concentration $0.1 \mu \mathrm{M})$. In all cases the bottom line corresponds to control injection of buffer. The binding kinetics of the experiments were too fast (Fuc-triazol) or too complex (multivalent) to evaluate dissociation constants and steady-state analysis was performed instead. PNA: Peptide nucleic acid; RSL: Ralstonia solanacearum lectin; SPR: surface plasmon 
gain with higher order oligomeric interactions. Nonetheless, the sub-nanomolar affinity for the hexameric assembly remains notable considering that fucose ligand by itself has an affinity of $2200 \mathrm{nM}$ (i.e. $>1000$ fold gain in affinity).

We next asked if the significant gain in affinity observed for the static dimeric assembly could be achieved with shorter PNA that would not form stable duplexes in solution but only in the presence of the lectin through the cooperative interaction of the PNA duplex and the ligand-target interactions (Figure 3). To this end, we prepared the same fucose ligand with selfcomplementary palindromic 4-mer PNA using two different
$2 \mu \mathrm{M}$; Figure S2-3). Thus at concentrations below $1 \mu \mathrm{M}$, the ligands $7 \mathrm{a}$ and $\mathbf{7 b}$ are predominantly in the dissociated monomeric form. However, the duplex should be stabilized by the high effective concentration of the PNA at the surface of the lectin and the lectin binding should be enhanced through the chelate effect of the hybridized PNA. As shown in Figure 3, while the fucose ligand has an affinity of $2.2 \mu \mathrm{M}$, ligand $7 \mathrm{a}$ has an affinity of $83 \mathrm{nM}$ whereas ligand $7 \mathrm{~b}$ has an affinity of $22 \mathrm{nM}$. When comparing the dissociation phase in the SPR sensorgrams, the fucose triazole adduct lacking the PNA has a very fast off-rate ( $k_{\text {off }}$ $=2 \times 10^{-1} \mathrm{~s}^{-1}$, Figure $\left.2 \mathrm{~B}\right)$, while the assembly arising from $7 \mathrm{~b}$

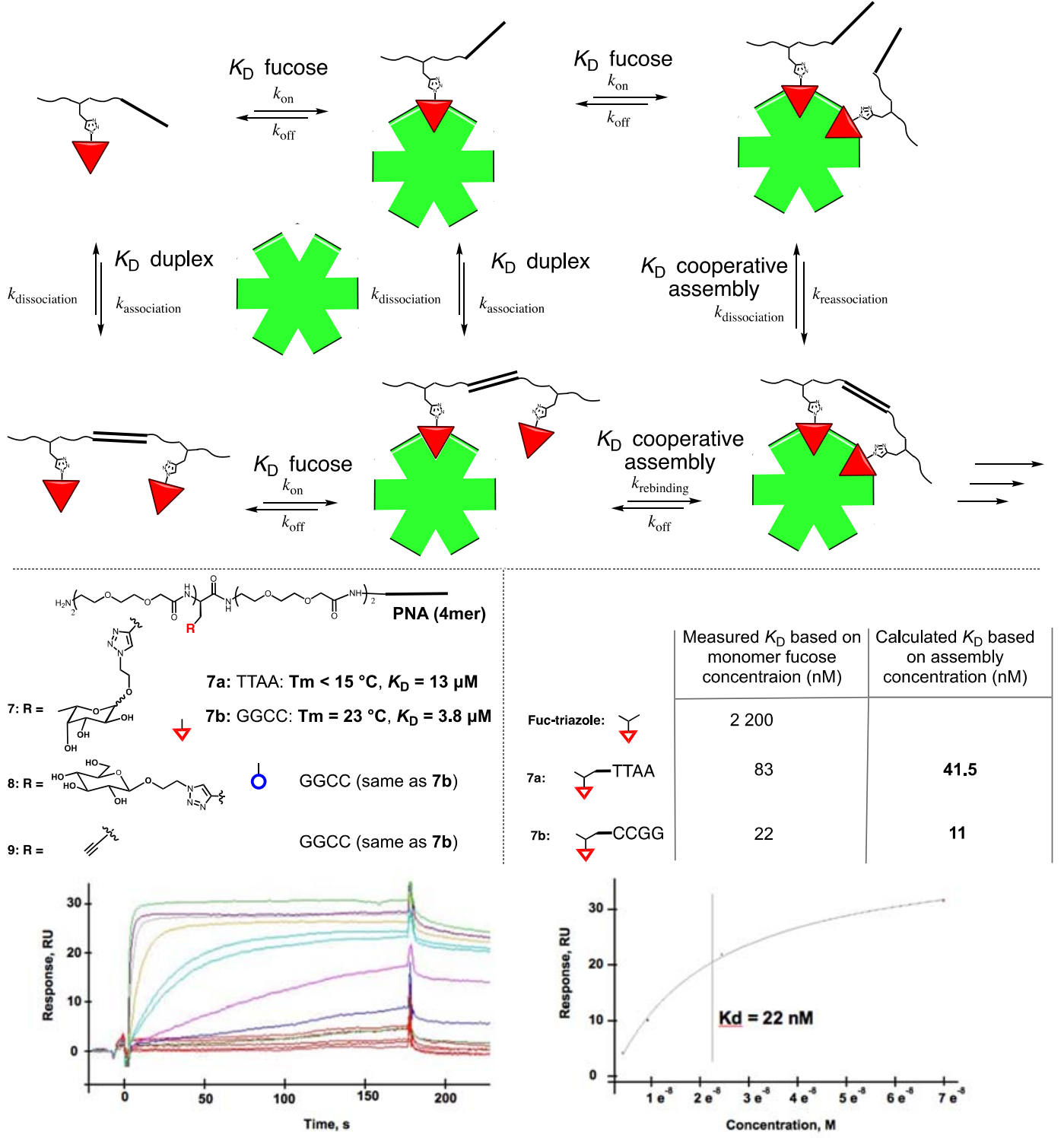

Figure 3. Top: Schematic representation of dynamic fucose dimer interacting with RSL; structure of compound 7-9 and affinities for assemblies prepared from 7a and 7b. Bottom: SPR sensorgrams for compound 7b injected on RSL chips with concentrations starting at $6 \mu \mathrm{M}$ and diluted by 3 -fold at each step and derivation of dissociation constant from steady state analysis. PNA: Peptide nucleic acid; RSL: Ralstonia solanacearum lectin; SPR: surface plasmon resonance.

sequences having different duplex stabilities (7a: TTAA: $K_{\mathrm{D}}=13$ $\mu \mathrm{M}, \mathrm{Tm}<15^{\circ} \mathrm{C}$ at $2 \mu \mathrm{M} ; 7 \mathrm{~b}: \mathrm{GGCC}: K_{\mathrm{D}}=3.8 \mu \mathrm{M}, \mathrm{Tm}=23^{\circ} \mathrm{C}$ at dissociates much slower ( $k_{\text {off }}=5 \times 10^{-4} \mathrm{~s}^{-1}$, Figure 3 bottom). We therefore observe a clear increase in the residence time at 
concentrations well below duplex formation with a very slow dissociation phase that is characteristic of multivalent interactions. As further controls, we prepared the equivalent of compound $\mathbf{7 b}$ with a glucose instead of fucose (8) or omitting conjugation with a carbohydrate (9). Neither of these controls had measurable affinities for the lectin (RSL). To further assess the cooperative dynamic assembly of $\mathbf{7 b}$, we performed the SPR measurements of $\mathbf{7 b}$ binding to RSL in the presence of increasing amount of control 9 which can compete for hybridization (up to 5 equivalents). The presence of control 9 had no impact on the affinity of $\mathbf{7 b}$ at stoichiometric amount (at this concentration, the probability of a $\mathbf{7 b}$ dimer from random hybridization is $25 \%$; Figure S1). At higher concentration ( 2.5 and 5 equivalents), the binding curves were slightly altered due to non-specific binding of $\mathbf{9}$ in the control channel, however, the same slow dissociation was observed as in the binding of $7 \mathbf{b}$ alone (at 5 equivalent of $\mathbf{9}$, the statistical probability of a $\mathbf{7 b}$ dimer is $2.7 \%$ ) suggesting a selfsorting of $\mathbf{7 b}$ dimer on the protein. The dramatic gain in affinity observed the dynamic assemblies of $\mathbf{7 a}$ or $\mathbf{7 b}$ as well as the lack of influence of $\mathbf{9}$ that can compete for hybridization can only be rationalized by the cooperativity of PNA hybridization and lectin interaction wherein the rebinding of ligand in the dimeric chelate is faster than PNA-duplex dissociation and likewise, duplex formation at the protein surface is faster than $k_{\text {off }}$ of the fucose ligand.

We then investigated the efficacy of the more potent assembly $\mathbf{7 b}$ to block binding of BambL to H1299 lung epithelial cells. For this purpose, we prepared both anomeric configurations of the fucose linked to the PNA. As shown in Figure 4, assemblies arising from both anomeric configurations were competent in inhibiting BambL binding to epithelial cells in a dose-dependent manner with an $\mathrm{IC}_{50}$ of $0.56 \mu \mathrm{M}$ and $0.94 \mu \mathrm{M}$ for the $\beta$-anomer and $\alpha$-anomer of fucose, respectively. Impressively, assembly $\mathbf{7 b}$ ( $\beta$ anomer) was 723 -fold more effective than the fucose alone. Assays with the Fuc-triazole lacking the PNA did not show any activity at concentrations up to $100 \mu \mathrm{M}$ and did not reach complete inhibition at the highest tested concentration (1 mM, Figure S4). The gain of efficacy observed in this assay with the dynamic assemblies concurs with affinity measurements on RSL. It should be noted that the assays were carried out at a RSL concentration of $178 \mathrm{nM}$ with a capacity to bind up to $1 \mu \mathrm{M}$ of fucose ligand. While occupancy of the six binding sites is not necessary for adhesion inhibition, the $\mathrm{IC}_{50}$ measurement points to the fact that the inhibition is achieved with nearly stoichiometric quantities of ligands relatively to the lectin. Furthermore, these cellular assays were performed at $37^{\circ} \mathrm{C}$, which further displaces the equilibrium away from duplex formation. The fact that assemblies $\mathbf{7 b}$ were more efficacious than the ligand alone at this temperature indicates that the cooperativity between ligand binding and duplex formation is still operative.

Multivalency plays a major role in biological processes and is omnipresent in the interaction of pathogenic microorganisms with their host. The search for high-affinity ligands that can inhibit these interactions has predominantly focused on oligomeric, polymeric and dendritic scaffolds functionalized with multiple ligand copies. ${ }^{[4,18]}$ To the best of our knowledge, this is the first example that demonstrates that such interactions can be inhibited with dynamic supramolecular assemblies in a cellular context. While dynamic combinatorial chemistry has been investigated for drug discovery and receptors using reversible covalent chemistry, ${ }^{[19-20]}$ the use of hybridization can be tuned to yield the benefit of a cooperativity between the ligand pairing through base pairing and chelate effect of the ligand's interactions with the target that is not achievable with covalent dynamic assemblies. This concept has inspired elegant studies to pair DNA- or PNA-encoded organic fragments for drug discovery. ${ }^{[21-26]}$ In the present case, the dynamic assembly was 723-fold more effective than the L-fucose alone in a cell-based assay. Many other pathogenic bacteria (e.g. P. aeruginosa via LecA or LecB, or uropathogenic $E$. coli via FimH) and bacterial products (e.g. Shiga toxin) that interact with host cell glycoreceptors could be in principle cleared by dynamic cooperative glycan assembly.
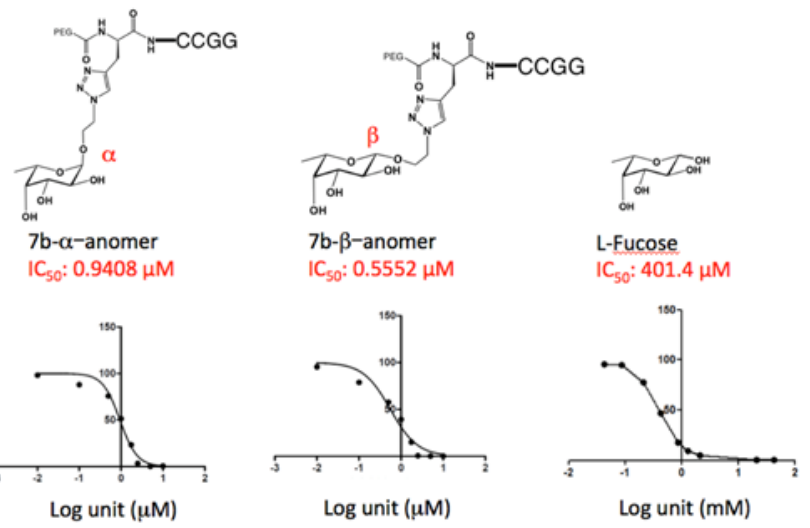

Figure 4. Inhibition of BambL binding to $\mathrm{H} 1299$ lung epithelial cells. Left: dynamic assembly arising from $\mathbf{7 b}$ with the fucose $\alpha$-L-fucose conjugate; Middle: dynamic assembly arising from $7 \mathbf{b}$ with the fucose $\beta$-L-fucose conjugate; Right: L-Fucose (corresponding bar charts with error bars are provided in Figure S4). BambL: Burkholderia ambifaria lectin

\section{Acknowledgements}

This work was supported by CNRS, Université Grenoble Alpes, Labex ARCANE (ANR-11-LABX-0003-01), Swiss National Science Foundation (SNSF) and the NCCR Chemical Biology for financial support. Al and WR acknowledge support by the French National Research Agency (ANR) and the German Federal Ministry of Education and Research (BMBF) in the framework of the EU ERASynBio project SynGlycTis (ANR-14-SYNB-0002-02; BMBF 031A464). WR was supported by the Excellence Initiative of the German Research Foundation (EXC 294), the Ministry of Science, Research and the Arts Baden-Württemberg (Az: 337532.20), and by a starting grant from the European Research Council (Programme "Ideas", ERC-2011-StG 282105). SZ acknowledges support from the China Scholarship Council.

Keywords: mutivalency • glycan $\cdot$ PNA $\cdot$ system chemistry

[1] C. Fasting, C. A. Schalley, M. Weber, O. Seitz, S. Hecht, B Koksch, J. Dernedde, C. Graf, E. W. Knapp, R. Haag, Angew. 
Chem. Int. Ed. 2012, 51, 10472-10498; Angew. Chem. 2012, 124, 10622-10650.

[2] A. Imberty, A. Varrot, Curr. Opin. Struc. Biol. 2008, 18, 567-576.

[3] T. Eierhoff, B. Bastian, R. Thuenauer, J. Madl, A. Audfray, S. Aigal, S. Juillot, G. E. Rydell, S. Muller, S. de Bentzmann, A. Imberty, C. Fleck, W. Romer, Proc. Nati. Acad. Sci. USA 2014 $111,12895-12900$

[4] A. Bernardi, J. Jimenez-Barbero, A. Casnati, C. De Castro, T. Darbre, F. Fieschi, J. Finne, H. Funken, K. E. Jaeger, M. Lahmann, T. K. Lindhorst, M. Marradi, P. Messner, A. Molinaro, P. V. Murphy, C. Nativi, S. Oscarson, S. Penades, F. Peri, R. J. Pieters, O. Renaudet, J. L. Reymond, B. Richichi, J. Rojo, F. Sansone, C. Schaffer, W. B. Turnbull, T. Velasco-Torrijos, S. Vidal, S. Vincent, T. Wennekes, H. Zuilhof, A. Imberty, Chem. Soc. Rev. 2013, 42, 4709-4727.

[5] A. Audfray, J. Claudinon, S. Abounit, N. Ruvoen-Clouet, G. Larson, D. F. Smith, M. Wimmerova, J. Le Pendu, W. Romer, A. Varrot, A. Imberty, J. Biol. Chem. 2012, 287, 4335-4347.

[6] N. Kostlanova, E. P. Mitchell, H. Lortat-Jacob, S. Oscarson, M. Lahmann, N. Gilboa-Garber, G. Chambat, M. Wimmerova, A. Imberty, J. Biol. Chem. 2005, 280, 27839-27849.

[7] J. Arnaud, K. Trondle, J. Claudinon, A. Audfray, A. Varrot, W. Romer, A. Imberty, Angew. Chem. Int. Ed. 2014, 53, 9267-9270; Angew. Chem. 2014, 126, 9421-9424.

[8] J. Arnaud, J. Claudinon, K. Trondle, M. Trovaslet, G. Larson, A. Thomas, A. Varrot, W. Romer, A. Imberty, A. Audfray, ACS Chem. Biol. 2013, 8, 1918-1924.

[9] K. Gorska, K.-T. Huang, O. Chaloin, N. Winssinger, Angew. Chem. Int. Ed. 2009, 48, 7695-7700; Angew. Chem. 2009, 121 7831-77836.

[10] K. T. Huang, K. Gorska, S. Alvarez, S. Barluenga, N. Winssinger, ChemBioChem 2011, 12, 56-60.

[11] M. Ciobanu, K.-T. Huang, J.-P. Daguer, S. Barluenga, O. Chaloin, E. Schaeffer, C. G. Mueller, D. A. Mitchell, N. Winssinger, Chem. Commun. 2011, 47, 9321-9323.

[12] C. Scheibe, A. Bujotzek, J. Dernedde, M. Weber, O. Seitz, Chem. Sci. 2011, 2, 770-775.

[13] F. Diezmann, O. Seitz, Chem. Soc. Rev. 2011, 40, 5789-5801.

[14] C. Scheibe, S. Wedepohl, S. B. Riese, J. Dernedde, O. Seitz, ChemBioChem 2013, 14, 236-250.

[15] A. Novoa, N. Winssinger, Beilstein J. Org. Chem. 2015, 11, 707719.

[16] S. Barluenga, N. Winssinger, Acc. Chem. Res. 2015, 48, 1319 1331.

[17] J. I. Yeh, B. Shivachev, S. Rapireddy, M. J. Crawford, R. R. Gil, S. C. Du, M. Madrid, D. H. Ly, J. Am. Chem. Soc. 2010, 132, 10717-10727.

[18] S. Cecioni, A. Imberty, S. Vidal, Chem. Rev. 2015, 115, 525-561.

[19] J. M. Lehn, Science 2002, 295, 2400-2403.

[20] O. Ramstrom, J. M. Lehn, Nat. Rev. Drug Discov. 2002, 1, 2636.

[21] S. Melkko, J. Scheuermann, C. E. Dumelin, D. Neri, Nat Biotechnol. 2004, 22, 568-574.

[22] K. I. Sprinz, D. M. Tagore, A. D. Hamilton, Bioorg. Med. Chem. Lett. 2005, 15, 3908-3911.

[23] J. P. Daguer, M. Ciobanu, S. Alvarez, S. Barluenga, N. Winssinger, Chem. Sci. 2011, 2, 625-632.

[24] G. Li, W. L. Zheng, Z. T. Chen, Y. Zhou, Y. Liu, J. R. Yang, Y. Y. Huang, X. Y. Li, Chem. Sci. 2015, 6, 7097-7104.

[25] F. V. Reddavide, W. L. Lin, S. Lehnert, Y. X. Zhang, Angew. Chem. Int. Ed. 2015, 54, 7924-7928; Angew. Chem. 2015, 127, 8035-8039.

[26] B. Shi, Y. Zhaou, Y. Huang, J. Zhang, X. Li, Bioorg. Med. Chem. Lett. 2016, 27, 361-369. 